\title{
L'horizon de la phénoménologie expérientielle : les formes incandescentes de la présence humaine
}

Jean Vion-Dury, Céline Balzani, Jean-Arthur Micoulaud-Franchi et Jean Naudin

\section{OpenEdition}

1 Journals

Édition électronique

URL : http://journals.openedition.org/alter/921

DOI : 10.4000/alter.921

ISSN : 2558-7927

Éditeur :

Association ALTER, Archives Husserl (CNRS-UMR 8547)

Édition imprimée

Date de publication : 1 novembre 2013

Pagination : 337-351

ISBN : 978-2-95-223749-9

ISSN : 1249-8947

\section{Référence électronique}

Jean Vion-Dury, Céline Balzani, Jean-Arthur Micoulaud-Franchi et Jean Naudin, « L'horizon de la phénoménologie expérientielle : les formes incandescentes de la présence humaine », Alter [En ligne], 21 | 2013, mis en ligne le 01 juin 2019, consulté le 06 juillet 2019. URL : http:// journals.openedition.org/alter/921 ; DOI : 10.4000/alter.921 


\title{
L'HORIZON DE LA PHÉNOMÉNOLOGIE EXPÉRIENTIELLE : LES FORMES INCANDESCENTES DE LA PRÉSENCE HUMAINE
}

\author{
Jean Vion-Dury, Céline Balzani, \\ Jean-Arthur Micoulaud-Franchi, Jean Naudin
}

\section{I) Introduction : la phénoménologie expérientielle.}

Depuis la plus haute Antiquité, et dans plusieurs continents, les philosophes, les religieux, les sages, les mystiques mais aussi d'autres humains n'ayant que la prétention de moins mal comprendre ce qu'il en est de leur vie mentale, posent un regard réflexif sur le contenu et l'organisation de la vie de l'esprit. En Occident, une étape décisive fut franchie par Husserl, quand il prit le parti d'une analyse systématique et scientifique des vécus conscients, en opposition avec toute la psychologie objectivante et scientiste de son époque.

Depuis, ainsi que l'indiquent Froese, Gould et Barett ${ }^{1}$, diverses formes d'analyse des vécus subjectifs ont vu le jour, dont la Programmation Neurolinguistique (PNL), la méditation et la pleine conscience, la Focalisation et la Pensée aux Limites, l'échantillonnage descriptif de l'expérience, l'Empathie et la Déduction. Parmi ces méthodes, l'entretien d'explicitation (EDE), proposé par Vermersch², situé au carrefour de la psychologie contemporaine et de la phénoménologie husserlienne, ouvre la voie d'une psychophénoménologie de l'expérience subjective. C'est sur cette dernière forme de méthode d'analyse des vécus subjectifs que nous nous focaliserons.

\footnotetext{
${ }^{1}$ Tom Froese, Cassandra Gould, et Adam Barett A, « Re-viewing from within. A commentary on first- and second person methods in the science of consciousness ", Constructivist Foundations, 6 (2), 2011, p. 254-269.

2 Pierre Vermersch, L'entretien d'explicitation, Issy-les-Moulineaux, ESF, 2010.
} 
L'EDE, bien que souvent tourné de manière pragmatique vers une analyse de l'action dans des contextes d'enseignement ou de remédiation à des difficultés cognitives, constitue une des modalités pratiques d'une phénoménologie de l'expérience vécue (phénoménologie expérientielle). Mais la phénoménologie expérientielle, comme phénoménologie pratique de la vie et exploration des vécus conscients réflexifs ou pré-réflexifs ${ }^{3}$, ouvre le chemin d'une double problématique. Outre l'exploration de la conscience, de ses contenus, de ses structures, de ses variations, dans la lignée de l'œuvre husserlienne, en même temps, et de manière reliée, comme l'autre face d'une médaille, la phénoménologie expérientielle ouvre la voie à l'exploration de l'Etre-là, du Dasein, de la Présence humaine dans la lignée de la pensée heideggérienne. Si la première direction d'exploration a été largement développée par les promoteurs de $\mathrm{l}^{\prime} \mathrm{EDE}^{4}$, la seconde a été peu détaillée.

\section{II) Style phénoménologique et phénoménologie expérientielle du second ordre.}

Dans un article récent abordant les possibles applications au champ médical de la pratique de la phénoménologie expérientielle en général et de l'EDE en particulier, nous avons insisté sur le fait que l'exercice de la phénoménologie générait une éthique spécifique ${ }^{5}$. En $\mathrm{d}^{\prime}$ autres termes, considérer la phénoménologie comme une pratique ${ }^{6}$ éventuellement quotidienne et ne pas la limiter à une approche théorique, conduit à la constitution progressive d'un style, assez reconnaissable chez les phénoménologues. Ce style phénoménologique, s'il admet des variantes chez les différents experts ou pratiquants de la phénoménologie expérientielle, intéresse en particulier la manière d'être en relation avec les autres, de se positionner dans le monde, et de s'ouvrir aux possibles de l'existence. On pourrait dire aussi, qu'au delà de l'expérience personnelle, de la culture phénoménologique, mais aussi de dispositions personnelles particulières sur lesquelles la

\footnotetext{
${ }^{3}$ Natalie Depraz, Francesco Varéla et Pierre Vermersch, À l'épreuve de l'expérience. Pour une pratique phénoménologique, Bucarest, Zeta books, 2011.

${ }_{4}^{4}$ Pierre Vermersch, Explicitation et phénoménologie, Paris, PUF, 2012.

${ }_{5}$ Céline Balzani, Jean-Arhur Micoulaud-Franchi, Norma Yunez, Anna Fagot, Anne-Sophie Mariaud, Chu-Yin Chen, Claire Maury Rouan, Maria-Laura Martin-Sentinelli, Jean Naudin et Jean Vion-Dury, «L'accès aux vécus pré-réflexifs : quelles perspectives pour la médecine en général et la psychiatrie en particulier ? », in Annales Médico-Psychologiques, no 171, 2013, p. 118-127.

${ }^{6}$ Natalie Depraz, Comprendre la phénoménologie, une pratique concrète, Paris, Armand Colin, 2006.
} 
psychologie cognitive ou clinique a peu travaillé, se découvre une modalité originale de la manière d'être conscient, et qui correspondrait à un type ou une variété d'intentionnalité.

Cette variété d'intentionnalité qui semble ainsi commune à ceux qui pratiquent l'exploration systématique et approfondie de leurs vécus conscients, se traduit par une intensité intellectuelle, émotionnelle et affective initialement nécessaire à la réalisation des accordages affectifs et à la qualité de l'écoute dans $\mathrm{l}^{\prime} \mathrm{EDE}^{7}$, mais qui en quelque sorte s'infiltre, se dépose et se structure à l'intérieur même de la manière d'être du phénoménologue, et y devient un habitus, c'est à dire tout à la fois lieu où l'on habite et habitude. Il nous semble que cette intensité a quelque chose à voir avec une incandescence, quelque chose qui est en feu ou qui brûle, et c'est ce que nous développerons dans les paragraphes suivants.

Dans cet article, nous allons tenter de caractériser ce style phénoménologique. La démarche que nous avons employée a consisté à réaliser une analyse phénoménologique de la manière dont les auteurs de ce texte ont vu leur habitus changer au cours de ce type de pratique phénoménologique. D'une part, cette analyse phénoménologique a porté sur ce qui se passe pendant les EDE, en particulier dans ce processus complexe d'intersubjectivité qui se constitue au cours de l'entretien, sur la création de cet espace commun de conscience dans lequel l'expérience de celui qui accède à ses vécus conscients est vécue par celui qui conduit l'entretien ${ }^{8}$. D'autre part, cette analyse porte sur ce que cela fait de voir son vécu conscient quotidien se transformer et comment cela change sa manière d'être au monde.

En pratique, nous n'avons pas réalisé d'EDE pour accéder aux caractéristiques des évolutions de nos habitus, mais nous avons, à de nombreuses reprises, partagé des auto-explicitations sur ces modifications de la manière d'être au monde et aux autres et réalisé des analyses de nos vécus conscientiels dans de nombreuses séances de travail utilisant le «discours en mode explicitant» (cf. plus loin). Ainsi, nous avons développé une sorte de phénoménologie expérientielle de la phénoménologie expérientielle, une méta phénoménologie expérientielle, c'est à dire une phénoménologie expérientielle $d u$ second ordre par référence à la pensée du second ordre de Von

\footnotetext{
${ }^{7}$ Cela serait aussi vrai d'ailleurs pour certains méditants ou mystiques.

8 «L'accès aux vécus pré-réflexifs : quelles perspectives pour la médecine en général et la psychiatrie en particulier? ", in Annales Médico-Psychologiques, op. cit.
} 
Foerster ${ }^{9}$, telle qu'elle est étudiée dans une épistémologie de la complexité.

\section{III) Le style phénoménologique et la présence.}

Si l'on peut interpréter ce qui se passe pendant un EDE sous la forme de partages de flux de conscience en lien avec l'accordage affectif, en revanche, sur la longue durée, la modification des habitus liée à la pratique régulière voire intense de la phénoménologie sous ce mode, l'apparition du style phénoménologique en fait, ne relève plus seulement de ce qui est en jeu dans le présent de l'entretien d'explicitation. C'est ainsi qu'on peut comparer l'entretien d'explicitation à une comète. Alors que la comète (l'entretien) reste localisée voire ponctuelle, elle est suivie d'une queue, beaucoup plus vaste à la fois en durée et en épaisseur. L'entrecroisement des "queues de comètes » lors de la pratique régulière de l'explicitation, ce que ces EDE changent en nous non plus au moment même de l'entretien mais dans son décours (et il n'est pas rare qu'un entretien fasse sentir ses effets plusieurs semaines ou mois après son déroulement), c'est cela qui conduit à ce nouvel habitus, et à un nouveau type de Présence.

La polysémie et la complexité des renvois référentiels de la notion de Présence dans les différentes langues ne nous permet pas ici de les détailler. Nous partirons de la traduction du Dasein heideggérien, traduit en français tout autant par l'« Être-là » que par Présence. On retrouvera chez Heidegger de nombreuses références à la Présence dans « Être et Temps »10, "Les problèmes fondamentaux de la phénoménologie $»^{11}$ et $~$ Acheminement vers la parole $»^{12}$. D’autres références comme l'article de Sa Cavalcante Schuback ${ }^{13}$ ou le livre de Gumbrecht ${ }^{14}$ permettront d'aller plus loin dans la compréhension de cette difficile notion.

D'emblée retenons quelques aspects fondamentaux du Dasein entendu comme Présence.

\footnotetext{
${ }_{9}$ Voir Evelyne Andreewsky et Robert Delorme, Seconde cybernétique et complexité. Rencontres avec Heinz von Foerster, Paris, L'Harmattan, 2006.

${ }^{10}$ Heidegger, Être et temps, Paris Gallimard, 1977.

${ }^{11}$ Heidegger, Les problèmes fondamentaux de la phénoménologie, Paris, Gallimard, 1975.

12 Heidegger, Acheminement vers la parole (1959), Paris, Gallimard, 1976.

${ }^{13}$ Marcia Sa Cavalcante Schuback, "La perplexité de la présence. Notes sur la traduction de Dasein », in Les études philosophiques, no 62 2002/3, p. 257-279.

${ }^{14}$ Hans Ulrich Gumbrecht, Éloge de la Présence. Ce qui échappe à la signification, Paris, Libella Maren Sell, 2010.
} 
Tout d'abord, la saisie de la présence relève de l'intuition parce qu'elle est donation dans le monde de la vie, lequel, comme le dit Husserl, est un domaine d'évidences originelles. Or les évidences du monde de la vie ont

«leur plus haute dignité dans la formation de la connaissance - plus haute que celle des évidences objectivo-logiques $\gg^{15}$.

De la Présence il n'est dès lors pas possible de réaliser une saisie ou une théorie scientifique, puisque la présentification d'un donné est du pur domaine de cette intuition.

Ensuite, en raison de son caractère intuitif, la saisie de la Présence nécessite d'une part une ouverture de notre champ perceptif dans l'ensemble de ses modalités sensorielles, et d'autre part que cette ouverture soit la plus grande possible et ressentie comme telle dans notre chair, un peu comme s'ouvrirait une immense antenne parabolique connectée à la totalité de nos terminaisons nerveuses. Cette ouverture doit être accompagnée d'une ad-tension et d'une intentionnalité spécifique en quelque sorte affectée d'un «coefficient d'accueil » de grande valeur.

Enfin la saisie de la Présence reste un processus atmosphérique, quasi évanescent, que $1^{\prime}$ on sent, en quelque sorte ${ }^{16}$. Dans cette attention spécifique à la Présence de l'autre, le langage, impuissant à décrire et à accompagner dans la modalité réflexive la saisie charnelle de cet horizon de la présentification, reste à l'état de balbutiement pour enfin, humilié, trouver lui-même son propre horizon qui n'est autre que le silence. À sa place, la musique, dans sa subtilité, sa ductilité et son infinie variété semble seule à pouvoir nous faire accéder à un entendement de la Présence.

À l'inverse les maladies sont également des possibilités de la Présence, qui devient alors gauchie. C'est ce qu'a proposé Binswanger, dans l'analyse existentielle ${ }^{17}$. Il parle à propos de la psychose schizophrénique de formes manquées de la Présence ${ }^{18}$. Mais, et cela est essentiel, ces formes manquées de la Présence constituent en fait des menaces générales immanentes à la condition humaine et qui ne sont pas spécifiques à la maladie mentale. Nous avons récemment

\footnotetext{
${ }^{15}$ Husserl, La crise des sciences européennes et la phénoménologie transcendantale, Paris, Gallimard, 1976, p. 145.

${ }^{16}$ Hubertus Tellenbach, Goût et atmosphère, Paris, PUF, 1983.

${ }^{17}$ Binswanger, Introduction à l'analyse existentielle, Paris, Éditions de minuit, 1971.

${ }_{18}$ Binswanger, Trois formes manquées de la présence humaine : la présomption, la distorsion, le maniérisme, Paris, Le cercle Herméneutique, 2002, p. 20 et 21.
} 
décrit également une forme manquée de la Présence dans le processus démentiel à savoir le double estompement qui réunit l'estompement de l'être-le-plus-propre et l'estompement de l'être-aumonde ${ }^{19}$.

C'est ainsi que, dans l'esprit de l'analyse existentielle de Binswanger, nous proposons une analyse des formes modifiées de la Présence survenant dans la pratique de l'EDE et dans les différents types de pratiques liées à la phénoménologie expérientielle. Cette modification de notre style personnel nous semble aller dans le sens d'une intensification, d'une exaltation (au sens étymologique de élévation, et non dans le sens plus contemporain d'agitation), et même d'une incandescence de la Présence au monde et aux autres.

Il nous semble que le terme $d^{\prime}$ incandescence convient particulièrement pour traduire à la fois ce processus d'accroissement $\mathrm{du}$ rayonnement personnel, mais en même temps de modification de l'énergie vitale qui s'accompagne de signes que l'on pourrait mettre globalement sous le vocable d'augmentation de la chaleur humaine.

Nous choisirons donc de décrire trois formes d'incandescence de la Présence. Le mot régime conviendrait tout autant en raison de sa connotation dynamique, mais le mot forme fait référence à la tradition de la Daseinanalyse à laquelle nous nous référons.

\section{IV) Première forme incandescente de la Présence : l'intersubjectivité phénoménologique ou l'intimité conscientielle.}

L'éthique de l'entretien d'explicitation requiert une syntonie et une empathie qui prennent le nom d'accordage affectif et qui utilisent des moyens de notre analyseur corporel pour saisir les infimes nuances de la prosodie et de la mimogestualité telle qu'elle est définie dans la pragmatique linguistique ${ }^{20}$ et que Stern dénomme les affects de vitalité ${ }^{21}$.

\footnotetext{
${ }^{19}$ Jean Vion-Dury, David Tammam, Céline Balzani, Jean-Arthur Micoulaud Franchi, Michel Cermolacce, Jean-Michel Azorin et Jean Naudin, "Phénoménologie des démences 2: "1'awarness" sans le "self" et le double estompement dans la maladie d'Alzheimer", Psychiatrie, Sciences Humaines et Neurosciences, 10 (2), 2012, p. 29-44.

${ }^{20}$ Jacques Cosnier, Le retour de psyché. Critique des nouveaux fondements de la psychologie, Paris, Desclée de Brouwern, 1998.

${ }^{21}$ Daniel N. Stern, Le moment présent en psychothérapie: un monde dans un grain de sable, Paris, Odile Jacob, 2004.
} 
Cet accordage affectif souvent intense entre les deux protagonistes d'un entretien d'explicitation réussi conduit à l'expérience particulière - et partagée - de la création d'une «bulle commune », espace commun de conscience dans lequel l'expérience de l'un est vécue par l'autre, non seulement comme une histoire qui serait racontée mais véritablement comme une histoire vraiment vécue désormais par celui qui ne l'a pas vécue. Dans ce cas, la matrice intersubjective ${ }^{22}$ dans laquelle toute relation humaine se construit, est non seulement mise en évidence, mais plus encore, intensifiée, densifiée.

La sensation d'une bulle commune, la prise de conscience de la matrice intersubjective intensifiée ouvrent le questionnement de la Présence de l'autre. L'expérience de co-Présence vécue dans cet espace intersubjectif se décrit avec la sensation (et le vécu) que l'on se tient dans l'expérience de l'autre, et d'une certain manière qu'on y est présent, qu'on s'y présentifie. Ceci est aussi vrai dans un groupe dont les membres participent, comme celui qui conduit l'entretien, au vécu de l'expérience de celui qui est interrogé. Il va s'agir en quelque sorte de la coalescence de deux (ou plusieurs) Dasein, de deux (ou plusieurs) Présences. Cette coalescence des Dasein donne accès à quelque chose qui relève de l'intimité de l'être-au-monde et de l'être-à-l'autre de chacun. Les espaces intersubjectifs ainsi construits entre les protagonistes de l'entretien (binôme ou groupe) délimitent cet espace de Présence dans lequel les affects de vitalités circulent, les énergies (au sens de ce qui fait mouvoir ou provoque le changement, energeia) s'échangent et dans lequel la relation intersubjective en quelque sorte s'exalte.

Dans cette matrice commune et dense ainsi formée, naît, par habitude, et en dehors de tout EDE, une manière d'échanger qui ponctue spontanément et fréquemment un discours «normal » de moments d'explicitation (ou d'auto-explicitation) des vécus conscientiels sur le mode du "comment», sans que jamais le cadre méthodologique de l'entretien ne soit convoqué. C'est ce que nous appellerons le discours en mode explicitant (DME). L'auto-explicitation des vécus pré-réflexifs devient également une seconde nature au point que l'habitus discursif du phénoménologue change, pour autant qu'il soit en présence d'un alter ego phénoménologue.

Ainsi, d'entretien en entretien, c'est la texture même des relations aux autres, en particulier des proches, qui se trouve à la fois dévoilée par la découverte de cette matrice intersubjective, de cette bulle commune d'intimité, et qui, en même temps, se trouve renforcée et

22 Idem. 
embellie. En particulier, cet accès réciproque à l'intime de l'autre dans les multiples EDE que nécessite la formation puis que tend à réitérer le plaisir de ce type d'introspection dans une pratique fréquente de la phénoménologie expérientielle, tout cela crée un type d'amitié inhabituelle, fondée non seulement sur l'estime, la sympathie, les intérêts communs, mais sur le partage d'une intimité qui ne se dévoile en réalité que là. Il s'agit de l'intimité du paysage intérieur de chacun, de sa modalité de Présence dans le monde, de la forme et de la structure de son régime d'intentionnalité. Contrairement à la psychothérapie et plus encore la psychanalyse, l'histoire de l'autre n'apparaît que comme le contexte accessoire de ses vécus de conscience et se dessine en creux sans que jamais il ne soit nécessaire d'en connaître le détail pour que cette intimité particulière, que nous qualifierons d'intimité conscientielle se développe ${ }^{23}$. En réalité dans cette présentification on saisit les traces des différentes ek-stases de l'être-été et de l'ad-venir, mais sans qu'une histoire événementielle n'ait à se déployer. Ce qui apparaît alors caractéristique de cette évolution des relations dans un groupe de travail de phénoménologie expérientielle, c'est la croissance très rapide de la confiance entre les membres du groupe. On pourrait dire que la pleine conscience (mindfulness) génère la pleine confiance (faithfulness).

Il est d'ailleurs frappant de constater à quel point la Présence de l'autre est ressentie au fond de nous même, dans notre corps, au moment de la création de la matrice intersubjective. Notre espace corporel qui trouve souvent un emplacement plus spécifique au centre de nous même s'ouvre d'une certaine manière et donne la sensation (pré-réflexive) d'une cavité (thoraco)abdominale vide et sans paroi antérieure. L'accueil de la Présence de l'autre ne se fait pas ainsi seulement dans notre regard, ou notre audition. Il se fait au fond de notre propre corps, sa voix pénétrant non pas seulement les oreilles, mais l'ensemble de notre chair, selon des trajets variables. Un sujet engagé dans une explicitation de ses vécus et à qui la question posée sur ce qu'est pour lui notre Présence, répondait: " Je vous sais. C'est tout noir mais c'est comme si c'était des filaments dorés, comme des contours. C'est tout noir, vos contours deviennent de plus en plus lumineux et j'ai des filaments qui viennent plutôt dans le ventre. J'ai une sensation d'enveloppement de votre présence ». Parler ici de la Présence n'est pas une conceptualisation théorique mais relève du

\footnotetext{
${ }^{23}$ Notons que l'expérience psychanalytique repose elle-même sur cette com-présence, en deçà du simple enchaînement historique des faits, et en ce sens l'interprétation participe d'une fonction poétique liée à la présence commune.
} 
rapport de comment se vit l'Incarnation de cette Présence de l'autre, son inscription corporelle en nous-même.

\section{V) Deuxième forme incandescente de la Présence : la modification de la conscience de soi et l'incandescence existentielle.}

Après plusieurs mois ou années de pratique de la phénoménologie expérientielle par le biais de l'EDE, le mode conscientiel du sujet qui la pratique change, dans la vie quotidienne.

Avant toute chose, il convient de spécifier que l'EDE, dans sa forme classique, n'est en aucune manière un moment isolé et enkysté dans des sessions spécifiques. Nous avons vu que l'EDE possède la structure d'une comète. Une fois terminé, l'entretien d'explicitation continue à agir dans la conscience réflexive et pré-réflexive du sujet et il n'est pas rare que des auto-explicitations plus ou moins élaborées ou des retours à cet entretien surviennent dans les jours qui suivent la session. L'entrelacs de «queues de comètes » transforme de cette manière en profondeur et progressivement l'habitus conscientiel du sujet.

Ainsi, d'EDE en EDE, avec leurs « queues de comète » et en passant par une pratique de plus en plus fréquente des auto-explicitations ${ }^{24}$, apparaît chez le phénoménologue une oscillation spontanée et rapide entre une conscience réflexive et un accès à la conscience pré-réflexive, en d'autres termes un passage rapide, spontané, conscient et assumé de «consciousness » à «awareness $»^{25}$. Cette oscillation amène fréquemment le sujet expert à «flotter» dans un flux de conscience intermédiaire assez proche d'une hypnose légère qu'il est assez aisé de transformer soit en un moment d'attention réflexive, soit au contraire en un laisser-aller consenti, assurant une commutation en une sorte d'auto-explicitation spontanée, relativement légère, qui, soit s'approfondit pour elle-même, soit se met à courir en parallèle avec une pensée réflexive dont elle devient une sorte d'ombre, d'arrière fond, comme deux vibrations parallèles et plus ou moins syn-

\footnotetext{
24 «L'accès aux vécus pré-réflexifs: quelles perspectives pour la médecine en général et la psychiatrie en particulier? ", in Annales Médico-Psychologiques, op cit.

${ }^{25}$ Natalie Depraz, La conscience: approches croisées des classiques aux sciences cognitives, Paris, Armand Colin, 2001. Dans notre texte, "awareness » est pris à la fois dans son sens neurophysiologique (le sentiment, le ressenti), mais aussi dans son sens de conscience ouverte dans lequel l'emploie N. Depraz.
} 
chrones, se propageant dans la même direction intentionnelle. Ce flux intermédiaire, cette sorte de veille généralisée, d'hypnose légère, donne globalement une conscience panoramique et maintient l'épochè.

Dès lors que le phénoménologue se laisse aller dans cette tendance à faire varier intentionnellement ou spontanément la qualité de son flux conscientiel, la pratique de l'auto-explicitation devient une seconde nature. Ainsi, de proche en proche, d'expériences d'introspection réalisées sur le mode de l'auto-explicitation en moments dédiés à des auto-explicitations de vécus, on en arrive à explorer sa vie consciente jusqu'en des profondeurs inattendues. C'est en quelque sorte une exploration de la structure intime de sa propre personnalité que le phénoménologue réalise ainsi. Il se dé-couvre et se dé-voile lui-même et pour lui-même dans son intimité existentielle, c'est-à-dire dans la vérité de son être. Il renoue avec des moments très anciens de l'enfance, il les revit, et ainsi peut, dans ce processus à la fois de réappropriation et de "plongée réflexive » que réalise chaque explicitation ${ }^{26}$, les mettre à distance et les intellectualiser.

Cheminant de la sorte dans le dévoilement de la vérité du Dasein, il découvre progressivement non pas des déterminations de son existence (ce qui supposerait une conception mécanique de notre mémoire) mais des replis de l'expérience dans lesquels, à certains moments il s'est logé ou caché. Il prend ainsi conscience de l'étant qu'il est comme mémoire cumulée, globale et intégrale ${ }^{27}$, désormais chaque fois en chaque instant. Car, « dès qu'il est, le Dasein a toujours nécessairement déjà été ${ }^{28}$.

Et se produit alors une sorte de transformation progressive de sa propre Présence, car il réalise, par le moyen de ces revécus en pleine conscience, des ek-stases de l'être et se saisit ainsi en tant qu'être-jeté dans le monde sur le mode de l'être-été ${ }^{29}$, prenant alors conscience de la disposibilité (l'humeur et les états d'âmes), de la peur et de l'angoisse qui s'étaient temporées dans $\mathrm{l}^{\prime}$ oubli ${ }^{30}$. Par l'auto-explicitation, le phénoménologue fait ainsi preuve d'authenticité et par là accepte d'être proprement lui-même dans le désir d'y voir clair-en-conscience.

Comment en effet, de telles prises de conscience, de tels dé-voilements de ce que la peur ou l'angoisse avaient laissé dans l'oubli ne pourraient-ils pas finalement, de proche en proche, changer la qualité

\footnotetext{
${ }^{26}$ Pierre Vermersch, «Conscience directe, conscience réfléchie », Intellectica, (2) 31, 2000, p. 269311.

${ }^{27}$ Bergson, Matière et mémoire, Paris, PUF, 2004.

${ }^{28}$ Heidegger, Les problèmes fondamentaux de la phénoménologie, op. cit., § 375 .

${ }^{29}$ Françoise Dastur, Heidegger et la question du temps, Paris, PUF, 2005, p. 61.

${ }^{30}$ Etre et temps, op. cit., p. 401.
} 
même de la Présence, élargir l'horizon de ses ek-stases, en accroître la densité et la richesse, et peut-être le rayonnement, et donner au Dasein un sentiment légitime d'ek-sistence plus intense, parce que ressenti comme accroissement des possibilités de son être-le-pluspropre? En ce sens, de même que naissant sous la flamme jaune qui fait reculer l'obscurité du lieu, la braise traduit l'incandescence de la bûche, de même l'accroissement du rayonnement et de la densité de la Présence, traduit une incandescence du Dasein qui, voyant clair-enconscience, accepte comme cadeau (présent) ou don du destin et le don de ce présent qui se présentifie. C'est en cela que l'on peut parler d'incandescence existentielle ${ }^{31}$.

Ce type d'expériences n'est cependant pas sans risque. Elles mettent à nu, comme si on atteignait ainsi, en enlevant les couches successives de l'oignon, le germe de ce bulbe. Et après d'intenses et fréquentes explicitations et auto-explicitations des vécus pré-réflexifs, il faut un certain temps avant que les couches protectrices ne se reforment et que la vie de la quotidienneté puisse se dérouler sinon comme avant, du moins à peu près «normalement ».

\section{VI) Troisième forme incandescente de la Présence : la modification de la conscience de l'Autre et l'embrasement relationnel.}

Les modifications profondes de l'éthos relatives à l'apparition du style phénoménologique, au travers de multiples expériences répétées d'EDE (qui sont en fait des moments d'épochè) conduisent, nous $\mathrm{l}^{\prime}$ avons $\mathrm{vu}$, à une conversion de notre manière de sentir le monde et de vivre notre relation à lui et à autrui.

$C^{\prime}$ est ainsi que nous pouvons décrire une troisième forme plus incandescente encore de la Présence qui procède à la fois de l'incandescence existentielle et de l'intimité conscientielle: il s'agit de l'incandescence relationnelle, que l'on pourrait aussi appeler amitié phénoménologique.

Cette amitié phénoménologique se construit donc et se développe à la faveur des accordages affectifs de plus en plus profonds et spontanés, qui font, qu'en réalité, les deux personnes qui rentrent ainsi en relation sont d'emblée dans un régime qui allie à la fois la

\footnotetext{
${ }^{31}$ C'est peut-être cette incandescence existentielle qu'a saisi La Tour dans La Madeleine Pénitente et Saint-Joseph Charpentier.
} 
réflexivité naturelle du discours de mode commun, un discours en mode explicitant, une auto-explicitation légère plus ou moins continue, et une explicitation quasi-continue, non verbalisée mais plutôt ressentie, de chacun par l'autre. C'est ainsi que d'une part se renforce une matrice intersubjective déjà très riche en raison de l'expérience commune et de l'intimité des protagonistes et, d'autre part, se crée une complicité immédiate de perception du monde, comme si la matrice commune se formant dès chaque rencontre était en réalité celle qui serait-au-monde, plutôt que celle de chacun séparément, dès lors qu'ils se sont (immédiatement) accordés. L'intimité conscientielle devient alors un Dasein commun, une possibilité inhabituelle de l'être-aux-autres, sorte de Présence commune au monde.

Le DME devient alors la forme quasi exclusive du dialogue et se généralise. D'ailleurs, dans cette relation on ne discute plus, on partage. Nous appellerons cette modification du discours, ce partage, l'entre-explicitation, en référence à l'« entre » (aïda inter et intra subjective) de Kimura ${ }^{32}$.

Dans l'amitié phénoménologique, la Présence de l'autre nous est véritablement intime. Il se produit quelque chose que l'on pourrait qualifier de «l'être-dans-l'autre » réciproque et bijectif. Ce n'est pas une intimité de la vie de couple par exemple, encore que l'on pourrait dire qu'une vie de couple requiert parmi d'autres choses cette Présence intime de l'autre en soi. Plus exactement, c'est une préoccupation. Dans cette pré-occupation, nous nous pré-occupons de l'autre dans un mouvement éthique de responsabilité très proche de ce que propose Lévinas ${ }^{33}$, par une réponse qui ne s'origine pas cette fois sur l'apparition du visage mais dans le ressenti de cette Présence dont on a vu en fait qu'elle impliquait le jeu subtil entre praesens et absens ou sur l'anticipation diffuse du besoin de l'autre. L'autre nous importe, ce qui veut dire deux choses: qu'il compte pour nous en même temps qu'il nous amène à lui. Il génère en nous une disposition de devancement, qui est un autre devancement que celui du Dasein, dans la mesure où il inter-esse (il est entre) des étants intra-mondains et ne relève pas forcément de l'ad-venir de l'être-le-plus propre, mais de son présent immédiat.

C'est alors que la Présence se vit comme la disponibilité pour l'autre, comme la coalescence du praesens et de l'absens, comme le- jamaisfaire-défaut quand bien même l'autre n'est plus en chair et en os dans

${ }^{32}$ Bin Kimura, L'entre. Une approche phénoménologique de la schizophrénie, Grenoble, Jérôme Millon, 2000.

${ }^{33}$ Levinas, Ethique et infini, Paris, Fayard, 1982. 
l'instant de la présentification, comme le repliement dans le présent même à la fois de l'avoir-été ensemble et de l'advenir commun, comme rétention jamais achevée et protention toujours active dans le main-tenant d'un arrière-fond du moment présent auquel l'intentionnalité ad-tentive peut toujours parvenir sans effort. La Présence de l'autre est là dans un lieu significatif de notre Présence à nous, ou plus exactement dans une contrée dans laquelle en chacun la pensée commune séjourne et chemine ${ }^{34}$. Comme Présence, c'est-à-dire saisi dans les horizons des ses présentifications communes, l'autre est là, sous la main. Et cette contrée commune mais différente qui est présente en chacun, dans le déroulement du temps s'accroît pour prendre une place de plus en plus grande ainsi que le signifie, dans un vieux couple, cette expression bizarre qui exprime que l'autre devient sa moitié. Au sens strict, cette com-plicité de l'amitié ou de la vie conjugale exprime bien que les Présences sont pliées ensemble, c'est-àdire que des directions communes des horizons de la présentification sont si fréquentes qu'un horizon commun représentant toutes les possibilités de présentification ne devient pas une exception, mais plutôt la règle. C'est ainsi que naît la sensation, unique, d'être compris exactement.

L'amitié phénoménologique est comme toutes les grandes amitiés vécues dans l'humanité : celle de Montaigne et la Boétie, celle de Fénelon et de Madame Guyon. C'est une relation incandescente, ou plus précisément, pour garder le caractère dynamique et expansif de cette présentification commune, un embrasement relationnel. Sa caractéristique est de ne pas se situer principalement dans l'action, ou la réflexion spirituelle ou philosophique, mais outre ces deux aspects, de faire une très large part à l'explicitation des vécus préréflexifs, au dévoilement réciproque de l'intimité de sa conscience, à cette méditation sur ce qu'est vivre et être incarné, à cet étonnement face à la richesse de la vie mentale réflexive ou préréflexive.

Cette incandescence relationnelle a à voir avec le «je-ne sais-quoi » relatif à l'attraction magnétique des âmes telle qu'elle a pu être développée dans les dialogues de Platon puis dans toute une littérature qui s'étend jusqu'au XVII ${ }^{\text {e }}$ siècle, aube de la modernité, c'est-à-dire de la civilisation de la signification. Nous retrouverons alors dans ce cheminement, les notions de sympathie, de corps des mystiques, d'étincelle de l'âme, d'entrevision etc. ${ }^{35}$. Nous dirons volontiers dans

\footnotetext{
${ }^{34}$ Acheminement vers la parole, op. cit., p. 163

${ }^{35}$ Benjamin Riado, Le je-ne-sais-quoi. Aux sources d'une théorie esthétique au XVIe siècle, Paris, L'Harmattan, 2012.
} 
cette optique, que l'amitié phénoménologique est une résonance magnétique.

C'est à cela que l'incandescence relationnelle, qui caractérise l'amour comme l'amitié, conduit. Et cette pré-occupation, ou cette occupation de notre horizon présentiel par la Présence de l'autre, conduit à une vibration commune et à distance des états de l'âme. Quand la distance est spatiale pour deux sujets vivant dans le même temps historique, des phénomènes de convergence immédiate des pensées surviennent, toujours étonnants, qui d'un seul coup placent l'autre au premier plan de notre conscience et nous font éprouver physiquement la coalescence des Présences. Quand la distance est temporelle, comme c'est le cas quand le compositeur que tel musicien a tant joué et fréquenté est mort depuis plusieurs siècles, il est n'est pas si rare que ce compositeur, au détour d'une de ses pièce que l'on joue, affirme sa Présence, et « dise » à l'interprète : «Oui c'est comme cela qu'il faut le jouer, continue ».

\section{VII) Conclusion : vers l'ardeur spirituelle.}

Dans ce texte, nous laisserons de coté, provisoirement, une quatrième forme incandescente de la Présence : l'ardeur spirituelle ${ }^{36}$. Ce chapitre délicat mériterait un long développement et fera l'objet d'une réflexion plus spécifique car il concerne l'intime, le je-ne-saisquoi et toute une série d'expériences spirituelles dont la phénoménologie n'a été qu'en partie élaborée par Heidegger ${ }^{37}$ lui-même, M. Henry, J.-L. Marion, E. Stein etc. Dans ces expériences, qu'il $\mathrm{s}$ 'agisse des expériences de pic (peak experiences), des expériences à proximité de la mort (near death experiences, NDE), des expériences mystiques ou de méditation, des expériences du lien non quantitatif et non rationnel avec le monde elle se trouve à nouveau au centre de la problématique de la Présence. Dans ces cas nous dirons que la Présence ardoie.

$\mathrm{Au}$ fond, comme nous l'avons à plusieurs reprises mentionné, la phénoménologie expérientielle est non seulement une philosophie de la conscience ou une ontologie dont les concepts trouvent une incarnation, elle est aussi, et peut-être d'abord, une éthique qui nous

\footnotetext{
${ }^{36}$ L'ardeur est ainsi comprise comme le plus haut degré de l'incandescence, de ardere, prendre feu, s'enflammer, se passionner. En français, le verbe correspondant est ardoir ou ardre.

${ }^{37}$ On rappelle qu'avant Être et temps, Heidegger a écrit, en 1920-21, Phénoménologie de la vie religieuse.
} 
arrache à la quotidienneté moyenne, estompe le «on » ou le «il » au profit $\mathrm{du}$ «tu» et, d'une certaine manière, fonde une conscience morale sur la vérité de l'Être, c'est-à-dire de la Présence de soi aux autres et des autres à soi. De la phénoménologie expérientielle se dégagent non seulement cette éthique et cette conscience morale qui l'englobe, mais également une anthropologie dans laquelle faire société se fonde dans une compréhension du Dasein (être de l'étant) de chaque homme au travers de l'intersubjectivité de l'être-aux et avec-les autres. C'est là que se fonde à notre sens la dignité de l'humain, quand elle n'est pas fondée - et là chacun est libre de penser cette fondation - sur la certitude d'être une créature issue de Dieu.

En d'autres termes, poser phénoménologiquement le problème de la Présence, à partir de la pratique expérientielle, à la fois nous amène à penser en profondeur l'intersubjectivité en dehors de toute théorie psychologique, mais plus encore, nous oblige à sortir de la civilisation de l'interprétation (qui est celle des théories psychologiques ou psychanalytiques) pour nous fondre dans un monde au sein duquel tout, et notamment nous-même, est Présence. C'est dans cette contrée que coalescent, malgré leurs différences, deux grandes civilisations de l'humanité : la civilisation chinoise taôiste, et la civilisation occidentale judéo-chrétienne. 